\title{
Universal flu vaccines: a shot at lifelong cardioprotection?
}

\author{
Bahar Behrouzi@ and Jacob A. Udell@
}

Meta-analyses and cardiovascular outcome trials are laying the foundation for seasonal influenza vaccines to be a mainstay in the prevention of cardiovascular disease, rivalling established measures such as statin therapy. In this Clinical Outlook, we highlight current and future approaches for the use of influenza vaccination to reduce the risk of atherosclerotic cardiovascular disease and heart failure.

Landmark meta-analyses of data from observational studies and small randomized controlled trials (RCTs) have underscored an association between influenza vaccination and sizeable reductions in mortality and adverse cardiovascular (CV) outcomes ${ }^{1,2}$. These findings have led to the instigation of several large trials to examine the effect of seasonal influenza vaccines on CV outcomes ${ }^{3-6}$. A 2021 meta-analysis comprising almost 240,000 patients with $\mathrm{CV}$ disease (CVD) reported that influenza vaccination was associated with a $28 \%$ and an $18 \%$ reduction in relative risk of all-cause and $\mathrm{CV}$ death, respectively, and a $13 \%$ reduction in relative risk of major adverse $\mathrm{CV}$ events over a median follow-up of 20 months, compared with placebo or no vaccine ${ }^{2}$. Subsequently, the multicentre IAMI $\operatorname{trial}^{3}$ reported that the administration of an inactivated influenza vaccine shortly after hospital admission for myocardial infarction (MI) or high-risk stable coronary artery disease was associated with a $28 \%$ reduction in risk of major adverse CV events, a $41 \%$ reduction in CV mortality and a $41 \%$ reduction in all-cause mortality compared with placebo. The efficacy of individual vaccine formulations in high-risk patients with CVD has yet to be established, but so far no differences have been found in cardiopulmonary outcomes between a high-dose trivalent and a standard-dose quadrivalent vaccine in patients hospitalized for acute MI or heart failure ${ }^{4}$.

Of note, the effect sizes seen so far with influenza vaccination for secondary prevention of CVD are similar to those of guideline-directed therapy with statins, $\beta$-blockers and angiotensin-converting enzyme inhibitors, which have been shown to reduce $\mathrm{CV}$ mortality by approximately
$20-25 \%^{7}$ (FIG. 1). At present, three large RCTs are evaluating the cardioprotective effects of influenza vaccination in different populations, including DANFLU-1 and IVVE ${ }^{5,6}$.

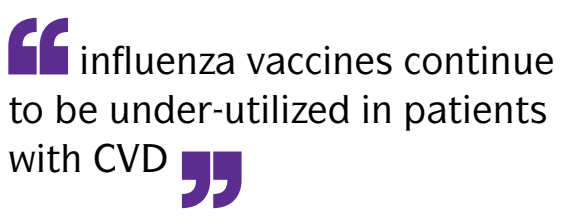

Despite the near-universal guideline recommendations for influenza vaccination in patients with CVD, influenza vaccines continue to be under-utilized in patients with $\mathrm{CVD}^{8}$. Alongside persistent vaccine hesitancy and misinformation, this under-utilization is attributable to the limited, platform-agnostic effectiveness of existing influenza vaccine strategies (approximately $40-60 \%$ protection against infection), which cannot facilitate rapid mass production of vaccines in response to new circulating mutations of influenza viruses ${ }^{8}$. This current strategy also underestimates the full cardioprotective potential of influenza vaccines - yet another argument in favour of fast-tracking the development of universal influenza vaccines to protect against all pandemic and seasonal influenza viruses.

In a first step towards the clinical development of a universal influenza vaccine, Nachbagauer and colleagues evaluated the safety and immunogenicity of a chimeric haemagglutinin-based universal influenza vaccine in healthy individuals with previous exposure to influenza infections and vaccinations. The universality of this vaccine platform relies on weaknesses inherent in the structure of the influenza virus.
All influenza viruses have two main glycoprotein spikes present on the viral envelope surface: haemagglutinin and neuraminidase. Traditional influenza vaccines elicit strain-specific neutralizing antibodies against the variable globular head domain of haemagglutinin, which demonstrates immunodominance (a phenomenon of immunity whereby a small number of specific peptides are selected as representative epitopes of a given antigen to the immune system for physiological efficiency). In contrast to the variable head, which is highly susceptible to antigenic drift and allows the virus to mutate and escape neutralization, the stalk domain of haemagglutinin, although immunosubdominant, is more conserved, and therefore holds promise as a vaccine target to elicit broadly cross-reactive antibodies. In a first-in-human study, a single administration of an adjuvanted, inactivated, split virion, chimeric haemagglutinin-based vaccine led to a safe, broad, robust and long-lived immune response in healthy young individuals, which persisted even 18 months after a booster dose $^{9}$. The antibody response also showed functional activities akin to those seen with protection from standard influenza vaccines, which indicates that these activities might also have a role in the protection induced by newer vaccine platforms. Looking to the future, the development of chimeric and mosaic haemagglutinin vaccines for other strains of influenza $A$ and influenza $B$ viruses are currently underway, with the ultimate goal of combining them into a trivalent vaccine against all drifted seasonal, zoonotic and pandemic influenza viruses.

The COVID-19 pandemic has also accelerated biotechnological investment in mRNA-based vaccine technology, while increasing public awareness of unconventional vaccine platforms. In 2021, Moderna enrolled 180 healthy adults into a phase I/II trial of a quadrivalent mRNA-based influenza vaccine, and began to test an mRNA-based respiratory syncytial virus (RSV) vaccine in a phase II/III trial that will involve an anticipated 34,000 participants ${ }^{10}$. Sanofi and Pfizer have also initiated phase I trials of their own mRNA-based influenza vaccines, given the advantages of this vaccine platform over conventional platforms, such as accurate strain selection and broader immune responses. These vaccines can also theoretically be 


\section{CLINICAL OUTLOOK}

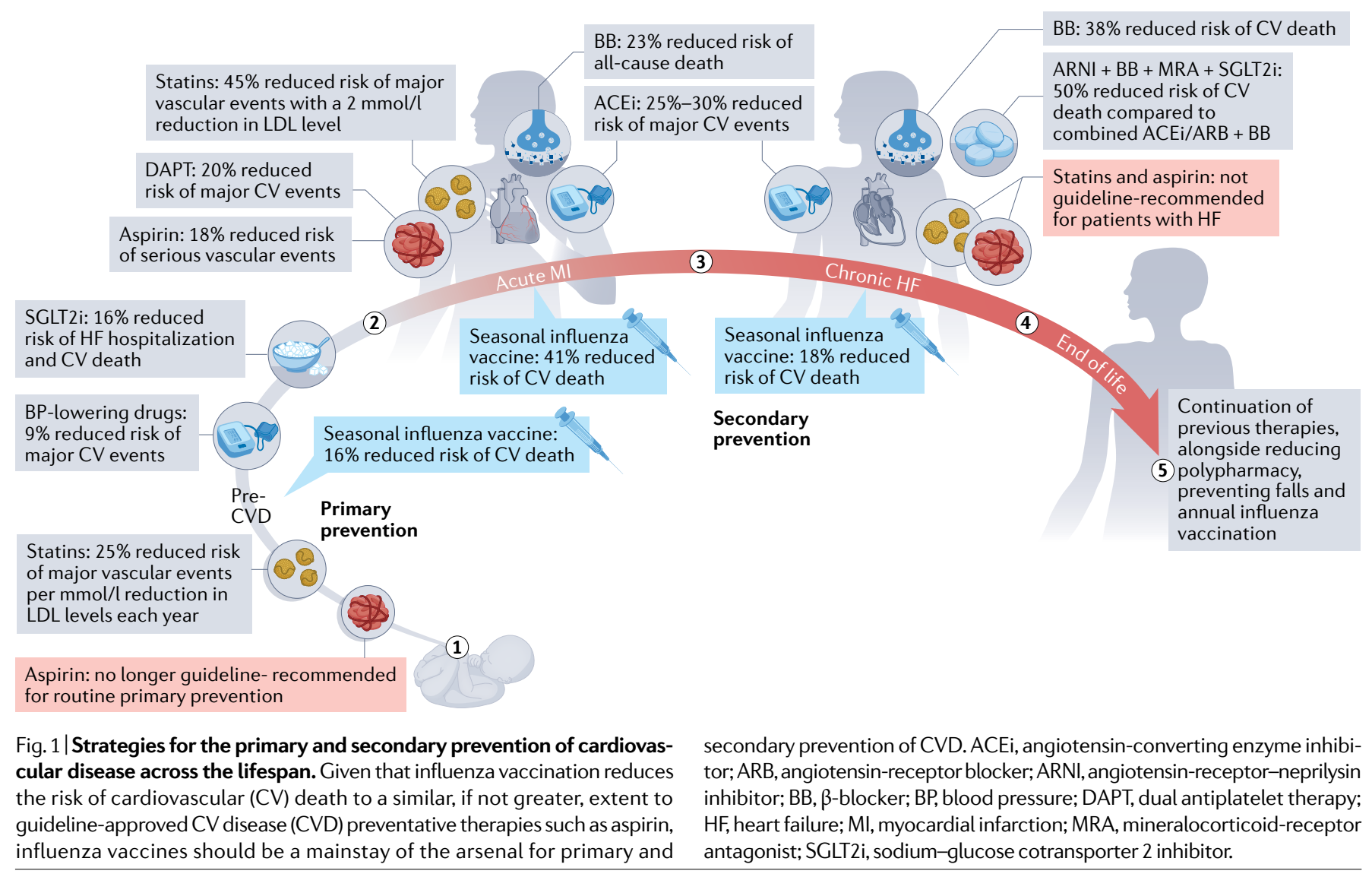

manufactured more quickly and easily combined into polyshots against various antigens, both within and across viruses. The potential downside of such an approach is patient tolerability, because post-vaccination symptoms tend to be more intense than with traditional vaccines. However, these transient, non-serious risks may be worth the benefit of protection against respiratory infections and associated CV events.

The same advances in vaccine platforms also hold promise for the future use of influenza vaccination for cardioprotection. First, the reported reductions in mortality and major $\mathrm{CV}$ events that result from the administration of technically 'subpar' traditional influenza vaccines are already sizeable. Therefore, boosting vaccine effectiveness for longer durations, reductions in antigenic evasion and increased coverage across more viral strains might translate to even larger effect sizes. Second, the COVID-19 pandemic has also underscored the vicious cycle between viral respiratory infections in general and cardiopulmonary diseases. Therefore, combining vaccines against influenza, RSV and severe acute respiratory syndrome coronavirus 2 (SARS-CoV-2) into a single polyshot is likely to increase the cardioprotective effects of the vaccine strategy.
To conclude, ongoing and future investigations of the efficacy, safety and cardioprotective effects of a myriad of universal vaccine platforms for influenza and other viruses are taking us one step closer towards the ideal of an effective vaccine to reduce the risk of CVD.

Bahar Behrouzi (D) ${ }^{1,2,3}$ and Jacob A. Udell (iD) 1,2,3,4凶 ${ }^{\prime}$ Cardiovascular Division, Department of Medicine, Women's College Hospital, Toronto, Ontario, Canada. ${ }^{2}$ Institute of Health Policy, Management, and Evaluation, Temerty Faculty of Medicine, University of Toronto, Toronto, Ontario, Canada. 3ICES, Toronto, Ontario, Canada.

${ }^{4}$ Peter Munk Cardiac Centre, University Health Network, Toronto, Ontario, Canada.

凶e-mail: jay.udell@utoronto.ca

https://doi.org/10.1038/s41569-021-00670-w

1. Udell, J. A. et al. Association between influenza vaccination and cardiovascular outcomes in high-risk patients: a meta-analysis. J. Am. Med. Assoc. 310, 1711-1720 (2013).

2. Yedlapati, S. H. et al. Effects of influenza vaccine on mortality and cardiovascular outcomes in patients with cardiovascular disease: a systematic review and meta-analysis. J. Am. Heart Assoc. 10, e019636 (2021).

3. Fröbert, O. et al. Influenza vaccination after myocardial infarction: a randomized, double-blind, placebo-controlled, multicenter trial. Circulation 144 , 1476-1484 (2021).

4. Vardeny, O. et al. Effect of high-dose trivalent vs standard-dose quadrivalent influenza vaccine on mortality or cardiopulmonary hospitalization in patients with high-risk cardiovascular disease: a randomized clinical trial. J. Am. Med. Assoc. 325, 39-49 (2021).
5. Hollingsworth, R. et al. Effectiveness of the quadrivalent high-dose influenza vaccine for prevention of cardiovascular and respiratory events in people aged 65 years and above: rationale and design of a real-world pragmatic randomized clinical trial. Am. Heart J. 237, 54-61 (2021)

6. Loeb, M. et al. Randomized controlled trial of influenza vaccine in patients with heart failure to reduce adverse vascular events (IVVE): rationale and design. Am. Heart J. 212, 36-44 (2019).

7. Michos, E. D. \& Udell, J. A. Am I getting the influenza shot too? Influenza vaccination as post-myocardial infarction care for the prevention of cardiovascular events and death. Circulation 144, 1485-1488 (2021).

8. Behrouzi, B. et al. Influenza vaccination to reduce cardiovascular morbidity and mortality in patients with COVID-19: JACC state-of-the art review. J. Am. Coll. Cardiol. 76, 1777-1794 (2020).

9. Nachbagauer, R. et al. A chimeric hemagglutininbased universal influenza virus vaccine approach induces broad and long-lasting immunity in a randomized, placebo-controlled phase I trial. Nat. Med. 27, 106-114 (2021).

10. Dolgin, E. mRNA flu shots move into trials. Nat. Rev. Drug Discov. 20, 801-803 (2021).

\section{Acknowledgements}

B.B. is supported by a University of Toronto MD/PhD studentship award and a Ted Rogers Centre for Heart Research Doctoral Award. J.A.U. is supported by Ontario Ministry of Research, Innovation and Science Early Researcher Award, as well as by the Women's College Research Institute and the Department of Medicine at Women's College Hospital and the University of Toronto.

\section{Competing interests}

B.B. has received grant support from Boehringer Ingelheim, Lilly and Sanofi Aventis. J.A.U. has received grant support from AstraZeneca, Novartis and Sanofi; has served as a consultant for Amgen, Boehringer Ingelheim, Janssen, Merck, Novartis and Sanofi; and has received honoraria from Boehringer Ingelheim and Janssen. 